\title{
Contribución de la educación superior a los Objetivos de Desarrollo Sostenible desde la docencia
}

\author{
Contribution of higher education to the \\ Sustainable Development Goals from teaching
}

\section{Débora Isabel Ramos Torres*}

DOI: $10.5944 /$ reec.37.2021.27763

Recibido: 24 de junio de 2020 Aceptado: 9 de noviembre de 2020

\footnotetext{
*Débora Isabel Ramos Torres: Doctora en Ciencias de la Educación. Maestría en Ciencias de la Educación Superior, Mención Docencia Universitaria y Sociólogo. Se desempeña en el programa de investigación del IESALC-UNESCO. Ha realizado acciones de gestión de recursos de información acerca de las Conferencias Regionales de Educación Superior en América Latina y el Caribe, de las redes institucionales e internacionales universitarias y de las Cátedras UNESCO. También realiza docencia e investigación en el nivel de postgrado y su línea de investigación y publicación es en el área de la educación superior, producción y productividad académica y redes de educación superior. Datos de contacto: e-mail: d.ramos-torres@unesco.org
} 


\title{
Resumen
}

Los Objetivos de Desarrollo Sostenible (ODS) se han arraigado en las instituciones de educación superior (IES) por su compromiso con la formación de las personas con competencias clave relevantes para abordarlos. El artículo examina cómo se ha configurado la docencia como la dimensión con mayor potencial para incorporar el desarrollo sostenible y, cómo junto a la investigación, es considerada una de las principales áreas de contribución al logro de los ODS, concretizada en la integración de estos objetivos a los planes de estudios de las titulaciones oficiales que, como acción formativa se realiza. A partir de la revisión del Informe de la Segunda Encuesta Mundial de la Asociación Internacional de Universidades sobre Educación Superior, Investigación y Desarrollo Sostenible, del Informe anual del Acuerdo sobre los ODS de la Alianza Global y, del Dosier de la Red Española para el Desarrollo Sostenible, se analizan por cada ODS las acciones relevantes de integración de estos Objetivos Globales en la función docente y las referencias a experiencias como casos de estudio. El análisis de los resultados muestra una alta variabilidad entre las universidades respecto al grado de abordaje de cada uno de los ODS y la tendencia a identificar como «trabajo bien establecido», el efectuado con el ODS 4, como prioritario desde la docencia. Los casos de estudio analizados muestran una significativa diferenciación respecto a los tipos de acciones que realizan y a sus tendencias. El uso de encuestas como las analizadas resultan insuficientes para observar el desenvolvimiento de la integración en los planes de estudios, hacen falta más experiencias como la desarrollada por REDS, así como plataformas en línea en las cuales los docentes expongan sus experiencias de rediseños curriculares e incorporación de los ODS a los planes de estudios y se mapeo de las nuevas titulaciones que vienen emergiendo.

Palabras clave: Objetivos de Desarrollo Sostenible (ODS); Integración; Curriculum; Docencia.

\begin{abstract}
The Sustainable Development Goals (SDGs) have become entrenched in higher education institutions (HEIs) for their commitment to training people with relevant key competencies to address them. The article examines how teaching has been configured as the dimension with the greatest potential to incorporate sustainable development and how, together with research, it is considered one of the main areas of contribution to the achievement of the SDGs, concretized in the integration of these objectives to the study plans of the official degrees that, as a training action, are carried out. From the review of the Report of the Second World Survey of the International Association of Universities on Higher Education, Research and Sustainable Development, the annual Report of the Agreement on the SDGs of the Global Alliance and the Dossier of the Spanish Network for Development Sustainable, each SDG analyzes the relevant actions of integration of these Global Objectives in the teaching function and references to experiences as case studies. The analysis of the results shows a high variability between the universities regarding the degree of approach of each of the SDGs and the tendency to identify as "well-established work", the one carried out with SDG 4, as a priority from teaching. The case studies analyzed show a significant differentiation regarding the types of actions they carry out and their trends. The use of surveys such as those analyzed are insufficient to observe the development of integration in the curricula, more experiences such as that developed by REDS are needed, as well as online platforms in which teachers present their experiences of curricular redesigns and incorporation from the SDGs to the curricula and mapping of the new degrees that are emerging.
\end{abstract}

Keywords: Sustainable Development Goals (SDGs); Integration; Curriculum; Teaching. 


\section{Introducción}

A partir de la adopción por la Asamblea General de las Naciones Unidas en 2015 del documento titulado «Transformar nuestro mundo: la Agenda 2030 para el Desarrollo Sostenible» y los Objetivos de Desarrollo Sostenible (ODS) se asiste al creciente interés en estas temáticas, apreciándose asimismo, el reconocimiento del papel crucial de la educación superior en el logro de esa Agenda.

El núcleo de esta Agenda lo constituyen 17 ODS y cada uno (Tabla 1) incluye un conjunto de metas específicas (169 metas en total) que poseen el potencial para transformar la sociedad y movilizar a personas y países; comprenden asuntos que van desde el abordaje de la desigualdad hasta cuestiones estructurales como el cambio climático, el crecimiento económico sostenible, la capacidad productiva, la paz y la seguridad y, el tema de contar con instituciones eficaces, responsables e inclusivas en todos los ámbitos. Asimismo, los temas que se abordan adoptan un enfoque dinámico para lograr la igualdad de género y reflejan de forma equilibrada las dimensiones económica, social y ambiental del desarrollo sostenible. También en la Agenda se indican medios de implementación en cada uno de los objetivos y se destina un objetivo específico a la alianza mundial, que aglutinará a los gobiernos, la sociedad civil y otras instancias en un enfoque integrado del desarrollo internacional en favor de las personas y el planeta (ONU, 2015).

Estos objetivos y metas son integrados e indivisibles y brindan orientación para la acción a fin de garantizar una vida sostenible, pacífica, próspera y equitativa a través de enfoques universales, transformadores e inclusivos (UNESCO, 2017). También incluyen una compleja gama de desafíos sociales, económicos, y medioambientales, que requerirán de trasformaciones en el funcionamiento de las sociedades y las economías, y en cómo interactuamos con nuestro planeta. Los gobiernos, la sociedad civil organizada, el sector privado, empresas, y las instituciones educativas y de investigación, incluyendo las instituciones de educación superior (IES), como actores esenciales para ayudar a la sociedad a enfrentar estos desafíos.

En este contexto se considera que la educación superior tiene un papel crucial para el logro de los ODS, dada su labor de generación y difusión del conocimiento y su preeminente situación dentro de la sociedad y, más aun, cuando se considera que todos los ODS basan sus argumentos en el conocimiento, solicitan nuevos conocimientos y sugieren cambios futuros con referencia a la creencia en la ciencia; por lo que, se considera que es una responsabilidad social de las IES llevar la ciencia tanto a los responsables políticos como a la sociedad en general.

Asimismo, la educación superior se ha identificado explícitamente en el objetivo (4.3) de la Agenda 2030, respaldado por la Declaración de Incheon y el Marco de Acción ODS 4- Educación 2030 (UNESCO, 2015) y se viene considerando como uno de los factores que permiten alcanzar todos los ODS, por los impactos que los ODS tienen en la educación superior, ya que estos son dobles.

Por un lado, los ODS están transformando la forma en que funcionan las instituciones de educación superior. Esto incluye, por ejemplo, enseñar sobre ellos, específicamente investigar u orientar en general a la institución a lo largo de la Agenda 2030. Se pueden encontrar ejemplos de cambios provocados por la Agenda 2030 dentro de la institución en algunos de sus planes estratégicos institucionales y, por otro lado, las IES están contribuyendo activamente al logro de los Objetivos Globales, nuevamente a través de la enseñanza, la investigación, la participación comunitaria y las iniciativas del campus; 
por lo que se infiere que es probable que ninguno de los ODS pueda cumplirse sin la implicación de este sector. Tanto es así que han sido identificadas tres formas en que la educación superior se manifiesta en los ODS: como un objetivo en sí mismo, como parte del sistema educativo en su conjunto y, por último, como un motor para el desarrollo (McCowan, 2019).

Tabla 1.

Los Objetivos de Desarrollo Sostenible (ODS)

\begin{tabular}{|c|c|c|c|c|}
\hline 1 & $\begin{array}{l}\text { Poner fin a la pobreza en todas sus formas en todo el } \\
\text { mundo. }\end{array}$ & & & \\
\hline 2 & $\begin{array}{l}\text { Poner fin al hambre, lograr la seguridad alimentaria } \\
\text { y la mejora de la nutrición y promover la agricultura } \\
\text { sostenible. }\end{array}$ & & & \\
\hline 3 & $\begin{array}{l}\text { Garantizar una vida sana y promover el bienestar para } \\
\text { todos en todas las edades. }\end{array}$ & & 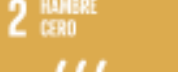 & 3 valisesiar \\
\hline 4 & $\begin{array}{l}\text { Garantizar una educación inclusiva, equitativa y de cali- } \\
\text { dad y promover oportunidades de aprendizaje durante } \\
\text { toda la vida para todos. }\end{array}$ & & & \\
\hline 5 & $\begin{array}{l}\text { Lograr la igualdad de géneros y empoderar a todas las } \\
\text { mujeres y niñas. }\end{array}$ & & ADAD & 6 Rgualupa \\
\hline 6 & $\begin{array}{l}\text { Garantizar la disponibilidad de agua y su gestión } \\
\text { sostenible y el saneamiento para todos. }\end{array}$ & & & \\
\hline 7 & $\begin{array}{l}\text { Garantizar el acceso a una energía asequible, segura, } \\
\text { sostenible y moderna para todos. }\end{array}$ & & & \\
\hline 8 & $\begin{array}{l}\text { Promover el crecimiento económico sostenido, inclusivo } \\
\text { y sostenible, el empleo pleno y productivo y el trabajo } \\
\text { decente para todos. }\end{array}$ & & 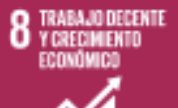 & UE \\
\hline 9 & $\begin{array}{l}\text { Construir infraestructuras resilientes, promover la in- } \\
\text { dustrialización inclusiva y sostenible y fomentar la inno- } \\
\text { vación. }\end{array}$ & & & \\
\hline 10 & Reducir la desigualdad en los países y entre ellos & ILLS & & \\
\hline 11 & $\begin{array}{l}\text { Lograr que las ciudades y los asentamientos humanos } \\
\text { sean inclusivos, seguros, resilientes y sostenibles. }\end{array}$ & & & \\
\hline 12 & $\begin{array}{l}\text { Garantizar modalidades de consumo y producción } \\
\text { sostenibles. }\end{array}$ & & & \\
\hline 13 & $\begin{array}{l}\text { Adoptar medidas urgentes para combatir el cambio } \\
\text { climático y sus efectos. }\end{array}$ & $13 \hat{n}$ & & 15 mina \\
\hline 14 & $\begin{array}{l}\text { Conservar y utilizar sosteniblemente los océanos, } \\
\text { los mares y los recursos marinos para el desarrollo } \\
\text { sostenible. }\end{array}$ & & & \\
\hline 15 & $\begin{array}{l}\text { Proteger, restablecer y promover el uso sostenible de } \\
\text { los ecosistemas terrestres, gestionar sosteniblemente } \\
\text { los bosques, luchar contra la desertificación, detener e } \\
\text { invertir la degradación de las tierras y detener la pérdida } \\
\text { de biodiversidad. }\end{array}$ & 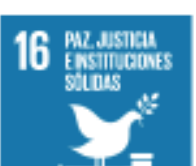 & 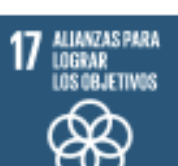 & $\begin{array}{l}\text { C9 } \\
\text { OBJETIVOS } \\
\text { DEDESARROLLO } \\
\text { SOSTENIBLE }\end{array}$ \\
\hline 16 & $\begin{array}{l}\text { Promover sociedades pacíficas e inclusivas para el desar- } \\
\text { rollo sostenible, facilitar el acceso a la justicia para todos } \\
\text { y construir a todos los niveles instituciones eficaces e } \\
\text { inclusivas que rindan cuentas. }\end{array}$ & & & \\
\hline 17 & $\begin{array}{l}\text { Fortalecer los medios de implementación y revitalizar la } \\
\text { Alianza Mundial para el Desarrollo Sostenible. }\end{array}$ & & & \\
\hline
\end{tabular}

Fuente: adaptado de Objetivos de Desarrollo sostenible [Infografía], de Naciones Unidas, 2015, https:// www.un.org/sustainabledevelopment/sustainable-development-goals/. CC By United Nations.

Progresivamente se ha reconocido la importante conexión entre la educación superior y la Agenda 2030 y del papel de las universidades, en el logro de los ODS. El texto de la 
Agenda menciona las funciones de las IES en nueve de los objetivos (ODS 2, 3, 4, 7, 8, 9, 13, 14 y 17) y, en alguna medida las universidades de todo el mundo están trabajando en todos ellos (IAU, 2020), aun así, estas instituciones son interpeladas por dichos objetivos, ya que, aún queda mucho por hacer para integrar plenamente la educación superior en los mecanismos establecidos para la consecución de la Agenda 2030; aunque ciertamente, la responsabilidad de implementar los ODS es expresamente ubicada a nivel de los gobiernos, la tarea de este nivel educativo queda más a nivel del compromiso con ellos.

Esto último se halla documentado en el trabajo publicado por el Instituto Internacional de la UNESCO para la Educación superior en América Latina y el Caribe titulado «Contribución de la educación superior a los Objetivos de Desarrollo Sostenible: marco analítico» (UNESCO-IESALC, 2020) en donde presenta un tratamiento teórico sobre la práctica de la sostenibilidad en las universidades, así como el abordaje de los ODS desde el ámbito de la investigación, pasando por los desafíos pendientes con la función formativa y con otras áreas del quehacer académico universitario. En este trabajo se deja planteada la necesaria continuidad investigativa de esta temática empleando otros enfoques y otras ideas y, fundamentalmente realizar demostración de avances y esfuerzos que desde las universidades se realizan.

\section{Integración global de los ODS en el curriculum: desafíos e implicaciones}

Dos son los desafíos que las universidades enfrentan en el marco de la Agenda 2030. Por un lado, formar profesionales capaces de proponer soluciones, fundamentadas en principios de sostenibilidad o en perspectiva de la Educación para el Desarrollo Sostenible (EDS) a los problemas que encontrarán en su desempeño profesional futuro y, por otro, el desafío de incorporar los Objetivos de Desarrollo Sostenible (ODS) en ese mismo proceso de formación como una palanca para trabajar todos los aspectos de la sostenibilidad y como contribución, al mismo tiempo, al alcance e implementación de estos objetivos (UNESCO, 2014).

Este último desafío se viene denominando Educación para los ODS (EODS) considerado como ese proceso de capacitación de las personas que han de implementar estos objetivos y, como acción realizada por y desde las universidades, EODS que viene siendo definida por la Red de Soluciones para el Desarrollo Sostenible (SDSN, por sus siglas en inglés) y la antena de esta, en España desde el 2015, la Red Española Desarrollo Sostenible (REDS) como el «... proporcionar a los estudiantes y al personal de la universidad los conocimientos, habilidades y actitudes necesarios para abordar los complejos desafíos del desarrollo sostenible, articulados por la Agenda 2030 a través de cualquier carrera o trayectoria vital que tomen» (Miñano y García, 2020, p. 6).

Sin embargo, desde el punto de vista teórico y técnico, el desafío de incorporar, introducir o integrar las dimensiones de los ODS en el curriculum universitario aún no parece suficiente (Albareda, et al., 2019; Paletta y Bonoli, 2019; Leal Filho et al., 2019; ValderramaHernández et al., 2019; HESI, 2019). El desarrollo de las competencias en sostenibilidad abordando los ODS desde la transversalidad o la especificidad, (Serrate et al., 2019; Segalàs y Sánchez, 2019) tiene complejas implicaciones que van desde la consideración a los propósitos globales e institucionales hasta aspectos de índole más particulares o técnicos que involucran a las expectativas y acciones de los principales actores del proceso de educación universitaria, como son el estudiantado y el profesorado. 
Este desafío implica, por un lado, asumir una propuesta educativa que promueva el cambio actitudinal y haga énfasis en la integración curricular de las problemáticas críticas que los ODS expresan (HESI, 2019; UNESCO, 2015, 2017) y por otra parte significa ir más allá de la sostenibilización curricular de las titulaciones universitarias (de grado y postgrado) como proceso de innovación de la práctica docente. Se trata, más bien, del abarcar no solamente la inclusión de contenidos de sostenibilidad en el temario de las distintas asignaturas, sino de promover, además, cambios más globales en la misma concepción del proceso educativo y de los procesos de enseñanza y aprendizaje (Leal Filho, 2019; Evans, 2019; Bautista-Cerro y González, 2017; Aznar et.al, 2016).

La creación de capacidades, a través de la educación, es un importante "medio de implementación» de los ODS (SDSN, 2017; UNESCO, 2015) y, por tanto, considerada una de las piedras angulares para su logro porque es esencial para alcanzar todos los objetivos (UNESCO, 2017). Una educación de calidad conduce a mejores resultados de desarrollo para las personas, comunidades y países, incluyendo mejor acceso al campo laboral, mejor nutrición y salud, ampliación de la equidad de género, mayor resiliencia frente a los desastres y, ciudadanos más comprometidos, entre otros beneficios.

La educación es valorada, por otra parte, como un medio crucial de apoyo y aceleración de la capacidad global para implementar los ODS. Así, las IES, a través de su extensa variedad de actividades y modalidades educativas y de aprendizaje (enseñanzas de grado y postgrado, prácticas profesionales, formación de adultos, educación a distancia y virtual, asociaciones estudiantiles) tienen un papel importante que jugar en este propósito de alcanzar los ODS desarrollando «soluciones sostenibles» a través de un plan de estudios que responda a estos objetivos globales (SDSN Australia/Pacífico, 2017).

Incorporar los contenidos, problemáticas, cualidades, contradicciones, principios y valores implícitos en los ODS dentro de los programas de estudio, incluyéndolos en sus planes de contenidos, como componentes indispensables de los procesos de formación en la educación superior, requerirá, asimismo, del abordaje de un conjunto de retos sociales y medioambientales que son multifacéticos y evidencian interconexiones complejas, incertidumbres y conflictos de valores.

Por añadidura, facilitar en los estudiantes el desarrollo de capacidades correctas y habilidades para pensar a través de la complejidad, de trascender los paradigmas, de aprender a través del diálogo y de la comunicación, de participar de una reflexión profunda, de generar una cosmovisión y valores, así como saber discriminar cuándo unas acciones apoyan el cumplimiento de los ODS y cuando otras, por el contrario, lo obstaculizan, así como otras importantes habilidades, conocimientos y experiencia profesional, se constituye en la gran implicación de este desafío.

$\mathrm{Al}$ equipar a los estudiantes con el conocimiento y las competencias que necesitan, no solo para comprender qué son los ODS, sino también para contribuir en calidad de ciudadanos informados a conseguir la transformación necesaria para lograrlos, se espera que los resultados de aprendizaje necesarios les permitan abordar los desafíos particulares de cada ODS facilitando de esta manera su consecución (UNESCO, 2017). Es decir, la incorporación de los estudiantes en un proceso de educación que comprende un conjunto amplio de saberes, valores, actitudes y habilidades vinculadas a los conceptos de sostenibilidad significa la utilización de los elementos integrantes de las llamadas competencias transversales de sostenibilidad relevantes (Sánchez y Murga, 2019; Rieckman, 2018; Mindt y Rieckman, 2017; UNESCO, 2017) y adecuadas para abordar los ODS y que ayudarán a crear implementadores más efectivos y acelerar la puesta en marcha de todos ellos (SDSN, 2017; UNESCO, 2017, 2015). 
En la educación superior los planes de estudios se configuran cada vez más en función de las competencias que los estudiantes deben desarrollar. La UNESCO (2017) define la competencias del siguiente modo:

«... atributos específicos que los individuos necesitan para la acción y la autonomía en distintos contextos y situaciones complejas. Incluyen elementos cognitivos, afectivos, volitivos y motivacionales. Por lo tanto, son una interacción entre el conocimiento, las capacidades y las habilidades, los intereses y las disposiciones afectivas. Las competencias no se pueden enseñar, sino que los mismos alumnos deben desarrollarlas. Se adquieren durante la acción, sobre la base de la experiencia y la reflexión». (p. 10).

La UNESCO (2017) también ha precisado las competencias clave a promover, ya que son consideradas como cruciales para el progreso del desarrollo sostenible, identificando los objetivos de aprendizaje y sugiriendo temas y enfoques pedagógicos importantes para cada uno de los 17 ODS. Estas competencias clave son entendidas como transversales, multifuncionales e independientes del contexto y poseen un alcance más amplio que las competencias específicas, ya que son consideradas necesarias para actuar de manera exitosa en las determinadas situaciones y contextos. (Tabla 2).

Específicamente, en la educación superior las competencias transversales introducen la responsabilidad y los principios éticos en las profesiones para que sean útiles a la sociedad y al planeta. Así, los ODS ofrecen una oportunidad a quienes tienen la responsabilidad de elaborar los diseños o rediseños de los planes de estudio de las distintas profesiones, para adaptarlos y concretar, definir y evaluar con claridad las nuevas competencias transversales. Según cual sea el ámbito profesional objeto de la formación, algunos ODS pueden ser más adecuados y próximos que otros, por lo que no es necesario asumir todas las metas, así como todas las competencias transversales, sino aplicarlas a los ámbitos propios de cada rama de conocimiento y de cada titulación (Cubero, 2019). 
Tabla 2.

Competencias transversales clave de sostenibilidad pertinentes a los ODS

\begin{tabular}{ll}
\hline Competencia de pensamiento & $\begin{array}{l}\text { Habilidades para reconocer y comprender las relaciones; para } \\
\text { analizar los sistemas complejos; para pensar cómo están integra- } \\
\text { dos los sistemas dentro de los distintos dominios y escalas; y para } \\
\text { lidiar con la incertidumbre. }\end{array}$ \\
\hline Competencia de anticipación & $\begin{array}{l}\text { Habilidades para comprender y evaluar múltiples escenarios } \\
\text { futuros, el posible, el probable y el deseable; para crear visiones } \\
\text { propias de futuro; para aplicar el principio de precaución; para } \\
\text { evaluar las consecuencias de las acciones; y para lidiar con los } \\
\text { riesgos y los cambios }\end{array}$ \\
\hline Competencia normativa & $\begin{array}{l}\text { Habilidades para comprender y reflexionar sobre las normas y } \\
\text { valores que subyacen en nuestras acciones; y para negociar los } \\
\text { valores, principios, objetivos y metas de sostenibilidad en un con- } \\
\text { texto de conflictos de intereses y concesiones mutuas, conocimiento } \\
\text { incierto y contradicciones }\end{array}$ \\
\hline Competencia estratégica & $\begin{array}{l}\text { Habilidades para desarrollar e implementar de forma colectiva } \\
\text { acciones innovadoras que fomenten la sostenibilidad a escala local } \\
\text { y más allá }\end{array}$ \\
\hline Competencia de colaboración & $\begin{array}{l}\text { Habilidades para aprender de otros; para comprender y respetar } \\
\text { las necesidades, perspectivas y acciones de otros (empatía); para } \\
\text { comprender, identificarse y ser sensibles con otros (liderazgo } \\
\text { empático); para abordar conflictos en grupo; y para facilitar la } \\
\text { resolución de problemas colaborativa y participativa }\end{array}$ \\
\hline crítico & $\begin{array}{l}\text { Habilidad para cuestionar normas, prácticas y opiniones; para } \\
\text { reflexionar sobre los valores, percepciones y acciones propias; } y \\
\text { para adoptar una postura en el discurso de la sostenibilidad }\end{array}$ \\
\hline lución de problemas & $\begin{array}{l}\text { Habilidad para reflexionar sobre el rol que cada uno tiene en la } \\
\text { comunidad local y en la sociedad (mundial); de evaluar de forma } \\
\text { constante e impulsar las acciones que uno mismo realiza; y de lidi- } \\
\text { ar con los sentimientos y deseos personales }\end{array}$ \\
\hline & $\begin{array}{l}\text { Habilidad general para aplicar distintos marcos de resolución de } \\
\text { problemas a problemas de sostenibilidad complejos e idear op- } \\
\text { ciones de solución equitativa que fomenten el desarrollo sostenible, } \\
\text { integrando las competencias antes mencionadas }\end{array}$ \\
\hline &
\end{tabular}

Fuente: adaptado de UNESCO. Educación para los Objetivos de Desarrollo Sostenible. Objetivos de aprendizaje. Copyright 2017 por la UNESCO.

La cuestión de la introducción de las competencias transversales de sostenibilidad en la educación superior ha sido abordada desde múltiples perspectivas: como presencia en los planes de estudio universitarios (Serrate et al.,2019; Segalàs y Sánchez, 2019; Gough y Longhurst, 2018; Murga-Novo, 2015), como distintas propuestas de competencias de sostenibilidad en la docencia universitaria (Mindt y Rieckman, 2017; Sánchez y Murga-Menoyo, 2019; Ng et al, 2019; García-González et al., 2019; Rieckman, 2018; Scarff y Ceulemans, 2017) y, también como investigación y práctica de la Educación Superior para el Desarrollo Sostenible (HESD).

Existen en este sentido, múltiples propuestas de enfoques pedagógicos para ofrecer una mejor «sostenibilidad en el curriculum» y se han desarrollado herramientas para ayudar a la enseñanza y el aprendizaje, de las cuales el Sustainability Literacy Test es un buen ejemplo, como plataforma en línea para IES, que facilita el acceso a conocimientos, habilidades y conciencia de sostenibilidad entre estudiantes y profesores, de tal forma que les motive a comprometerse profundamente con la construcción de un futuro sostenible tomando decisiones informadas y efectivas. También se emplea para evaluar las competencias clave de sostenibilidad (Sulitest, 2019). 
Sin embargo, la investigación sobre los enfoques pedagógicos y el impacto de las competencias sobre sostenibilidad es aún muy reducida (Lozano et al, 2017). De igual manera, faltan sistematizaciones de aquellas experiencias que reporten cómo desde la propia práctica docente se promueve en las aulas, en la perspectiva de una HESD o bajo el enfoque de una Educación para los ODS (EODS), la localización o ubicación de las evidencias de contribución por y en cada uno de los ODS.

No obstante, ya se observan avances de inclusión en las bases de datos especializadas de literatura dedicada a reportar investigaciones sobre la integración de los ODS en el desarrollo académico y profesional reportando casos de aplicación de innovaciones en las prácticas de enseñanza para mejorar las competencias de los profesores y desarrollar una educación de gestión responsable bajo el enfoque de sostenibilidad, así como la realización de procesos de rediseño curricular de los planes de estudio de educación superior (Nhamo y Mjimba, 2020; Shava et.al, 2020; Kanapathy et. $a l$, 2019). También se ubican reportes de casos inspiradores de educación para los ODS en las universidades, considerados como experiencias de docencia formal que muestran iniciativas de actividades formativas integradas en planes de estudio de titulaciones oficiales, orientadas a estudiantes universitarios (Miñano y García, 2020).

\subsection{Acciones específicas: los ODS en la docencia}

Uno de los aportes que los ODS ofrecen a las IES en general y universidades en particular que se comprometen con la consecución de ellos, es que amplían las oportunidades para formar personas con habilidades transversales y competencias clave relevantes para abordar todos los ODS, así como con aquellas capacidades profesionales y personales que los actuales y futuros ejecutores y responsables de implementar estos objetivos requieren.

Esta oportunidad representa, asimismo para las IES, no solo la posibilidad de formar en profesiones o trabajos que existen hoy, sino también para los que existirán en el futuro. Así, en ese proceso las IES comprometidas bajo un enfoque de cambio transformacional, estructuran o reestructuran cursos/asignaturas o diseñan programas de aprendizaje conducentes a titulaciones, de grado y/o postgrado (maestrías y doctorados), donde utilizando el marco de los ODS y la identificación de soluciones para los desafíos que estos implican, enseñar a profesionales con una sólida formación que puedan tomar decisiones con criterios de sostenibilidad.

El desarrollo y establecimiento de estos programas y cursos en los que han sido integrados los ODS se ha venido instaurando progresivamente. Sin embargo, la literatura muestra que el cambio institucional y la incorporación de nuevo contenido en las ofertas de formación puede ser un proceso lento y engorroso, que en muchos casos puede perdurar años (GUNi, 2019). No obstante, las IES avanzan y algunas introducen los ODS en el programa de, al menos, una asignatura en cada titulación, ya sean titulaciones de carácter oficial o propio (como en algunos países se suelen denominar).

También estos avances respecto a las transformaciones de las titulaciones y de los planes de estudio que los ODS hayan impactado, son difíciles de conocer con la debida precisión ya que, pocas universidades sistematizan sus procesos y comunican lo están haciendo. Aun cuando se conocen ya algunas experiencias de IES que han comenzado a mapear sus acciones y actividades relacionadas con los ODS tal como la Universidad de Costa Rica y la Universidad de Bolonia (IAU, 2020), sin embargo, en dichos mapeos aún no se especifican estos tipos de transformaciones. 
Con el propósito de analizar avances de las acciones que por cada ODS se pueden visualizar como experiencias de integración en la función docente, se revisan, en forma global, informaciones y datos obtenidos a partir del informe de la International Association of Universities (IAU) acerca de los resultados de la segunda edición de la Global Survey Report on Higher Education and Research for Sustainable Development (IAU, 2020), del Annual SDG Accord Report 2019 presentado ante el United Nations High-level Political Forum, elaborado por la Alianza Global de un grupo de redes de sostenibilidad de universidades e instituciones de educación superior de distintas partes del mundo (SDG Accord, 2019). Seguidamente se extraen algunas evidencias concretas como referencias a casos de estudio, examinando sus tendencias en cuanto a abordajes y tipo de acciones emprendidas, reforzando las experiencias referenciadas con las identificadas en el Dosier de la Red Española para el Desarrollo Sostenible (Miñano y García, 2020).

\section{Informaciones y datos de las Encuestas}

\subsection{Encuesta Global de la IAU sobre HESD 2019}

La Encuesta mundial de la IAU no pretendió examinar detalles sobre qué y cómo las universidades en forma particular se comprometen con los ODS, sino que sus resultados proporcionan una valiosa visión general de lo que está sucediendo en la educación superior y la investigación para el desarrollo sostenible en todo el mundo con énfasis en los ODS.

Habiendo sido distribuida a más de 7.894 instituciones del total (19.400) de IES registradas en la Base de datos de educación superior mundial (WHED) de la IAU, la encuesta recibió 536 respuestas válidas de 428 universidades de 101 países, representando un $7 \%$ de tasa de retorno. Las respuestas representan todas las regiones del mundo a saber: un 37 \% de respuestas provenientes de Europa, 23 \% de América Latina y el Caribe, $18 \%$ de África, $15 \%$ de Asia y el Pacífico, $4 \%$ de América del Norte y $3 \%$ de Oriente Medio. El $45 \%$ de los encuestados ocupan posiciones de liderazgos en sus universidades, otro $45 \%$ es personal académico o administrativo y el $10 \%$ restante son estudiantes (IAU, 2020).

La encuesta estuvo conformada por 35 preguntas distribuidas en cuatro partes en las que se abordaron temas sobre la educación superior y los ODS, los enfoques institucionales de la Educación para el Desarrollo Sostenible; las redes para el desarrollo sostenible; e informes sobre los obstáculos que IES enfrentan en su contribución a los ODS. Entre las interrogantes analizadas en el informe nos enfocamos a las referidas a la diferencia que las IES dan en el abordaje de los ODS y las que se refieren a las acciones que las universidades están haciendo para integrar los ODS al ámbito de los planes de estudio.

El principal de los resultados, presentados en la Encuesta mundial de la IAU que encontramos, es que la mayoría de las personas en las IES de las regiones son, al menos conscientes del hecho de que existen los ODS y que se requieren medidas urgentes para las generaciones futuras, habiéndose producido un cambio visible en la comprensión en general respecto a los temas del desarrollo sostenible.

La respuesta acerca del conocimiento percibido sobre la Agenda 2030 y los ODS ha aumentado, mientras que el conocimiento sobre educación para el desarrollo sostenible se mantuvo igual con respecto al año 2016, cuando se aplicó la primera encuesta de este tipo. Se destaca un hallazgo interesante, ya que entre los encuestados en 2019 
el conocimiento sobre los ODS es significativamente mayor que el que se tiene sobre la Agenda 2030, lo cual llama también mucho la atención por cuanto los ODS son esencialmente una parte de la Agenda 2030 y no existen sin ella; indicando esto, que aun cuando los ODS son una herramienta útil para promover el desarrollo sostenible, aún muchos no establecen, en detalle, sus conexiones.

Otro de los resultados atrayentes es cómo la adopción de los ODS aumentó en las IES a nivel mundial el interés acerca del desarrollo sostenible, encontrando que el $64 \%$ de los encuestados señala que la adopción de los ODS aumentó en su institución el interés sobre el desarrollo sostenible. Del mismo modo, el conocimiento sobre la Agenda 2030 y los ODS mejoró visiblemente en las IES. Sin embargo, algunas de las expresiones de los encuestados dejaron evidencias del cuestionamiento acerca de la naturaleza de los ODS y sobre su tratamiento o dudas sobre cómo trabajar con ellos, tal como, la opinión que enseguida se reproduce aquí: «Tenemos claro que queremos trabajar [con los ODS]; ahora no sabemos si el alcance propuesto dentro de las actividades educativas será el adecuado $^{1}$ (IAU, 2020, p. 23).

En el informe que da cuenta de la Encuesta, se reporta que las universidades de todo el mundo están trabajando en alguna medida en todos los ODS e indican los encuestados que sus instituciones están trabajando en al menos uno de los 17 ODS. El trabajo adopta distintas formas y focalizaciones, es decir, unos objetivos son más abordados que otros, recibiendo unos mucha atención y otros menos; así como también, se mantienen áreas de trabajo prioritarias en cada IES y, se abordan de muchas maneras diferentes según el área o ámbito de actuación; esto es, en la docencia (enseñanza y aprendizaje), la investigación, la participación comunitaria y las iniciativas del campus. Siendo estas, las misiones de las universidades como dimensiones clave para incorporar un enfoque de toda la institución o enfoque integral institucional con el que la Encuesta operó. A los efectos del propósito de este artículo hemos focalizado en los resultados del trabajo de las IES con los ODS en el área de la docencia.

Antes de continuar analizando los principales resultados de la Encuensta de la IAU y extraer el cómo las IES integran los ODS en la docencia, cabe puntualizar una advertencia que se señala en el informe y, que consideramos importante reproducir aquí. Esta advertencia se refiere a que los resultados obtenidos expresan el compromiso de las IES con cualquiera de los ODS y muestran un orden específico, así como preferencias y ámbito de trabajo particulares según los criterios de los participantes de la Encuesta; por lo que se trata de resultados de naturaleza subjetiva, dada la metodología empleada para la obtención de la información.

Por consiguiente, sus resultados pueden ser distintos a los arrojados por otros estudios que empleen o hayan empleado otros enfoques y metodologías diferentes, tal como los estudios de tipo bibliométrico. Además, las respuestas son subjetivas porque no muchas universidades a nivel mundial asignan su trabajo de acuerdo con los ODS, aun cuando ya se inicia a observar un número creciente que ha comenzado hacerlo, como veremos más adelante en la Tabla 5 .

Para clasificar el nivel de compromiso de la IES con cada ODS, se utilizaron dos categorías a saber: la categoría «trabajo bien establecido» para representar que la IES ya está trabajando en este objetivo de manera efectiva y, la categoría «trabajo emergente», para indicar que la universidad tiene el propósito proyectivo de aumentar el trabajo en esta área. (Tabla 3).

$1 \quad$ Traducción libre de: "We are clear that we want to work [with the SDGs]; now we do not know if the proposed scope within the educational activities will be adequate." 
De acuerdo con el Informe de la IAU, el ODS 4: Educación de calidad es el ODS con el que participan la mayoría de las universidades, seguido de cerca por el ODS 5: Igualdad de género y el ODS 13: Acción por el clima. Los ODS menos abordados son los ODS 14: Vida submarina, ODS 2: Hambre Cero y ODS 12: Producción y consumo responsables.

El área de trabajo que recibió más respuestas fue la docencia, seguida de cerca por la investigación, lo que indica que esta es la dimensión de la educación superior donde los encuestados ven el mayor potencial para incorporar el desarrollo sostenible. Así, se confirma que el ODS 4: Educación de calidad es una prioridad para las IES, por ser el objetivo más abordado, desde la enseñanza, con casi un $85 \%$ de encuestados indicando tener un «trabajo bien establecido» para lograr dicho objetivo. Además de ser considerado un objetivo particularmente relevante por comprender la meta ODS $4.7^{2}$, que se centra principalmente en la educación para el desarrollo sostenible, siendo esta meta transversal, no solo relacionada con el ODS 4, sino con otros de los 17 Objetivos globales, convirtiéndose así en uno de los objetivos más importantes, pero también más difíciles de medir, por la diversidad de temas que aborda.

Le sigue de cerca el ODS 5: Igualdad de género, que recibe el segundo lugar respecto al alto nivel de atención en el ámbito de la docencia, con un «buen trabajo establecido» y considerado también, junto al ODS 4 un objetivo transversal de importancia para alcanzar los otros objetivos. Finalmente, el tercer lugar de los objetivos más desarrollados en enseñanza y aprendizaje, con un «buen trabajo establecido», le corresponde al ODS 3: Salud y bienestar, ya que la educación superior es responsable de la capacitación de médicos, enfermeras y otros profesionales calificados de la salud (UNESCO, 2018) y en el que las IES, están contribuyendo a proyectos de conocimiento abierto, facilitando el acceso a la información y el intercambio de aprendizajes e investigaciones sobre enfermedades.

También los encuestados indicaron una alta disposición para aumentar el trabajo en otras áreas, en particular, el ODS 12: Producción y consumo responsables, es el ODS que recibe la mayor «nueva atención», ya que cuenta con el mayor número (34 \%) de respuestas de «trabajo emergente». Seguido del ODS 11: Ciudades y comunidades sostenibles (28\%), el ODS 9: Industria, innovación e infraestructura (26\%) y el ODS 1: Fin de la pobreza (26\%)

El objetivo que menos se aborda cuando se trata de enseñanza y aprendizaje es el ODS 14: Vida Submarina. Solo 14 \% de los encuestados indicaron realizar «trabajo bien establecido». El ODS 2: Hambre Cero, aunque actualmente es el menos desarrollado (12 \%), supera el ODS 14 cuando se trata del «trabajo emergente» (22 \%). Finalmente, el ODS 16: Paz, justicia e instituciones sólidas, es otro objetivo mencionado en la meta 4.7 del ODS 4 y nuevamente se considera transversal para todos. De igual forma es el cuarto objetivo más desarrollado en la sección sobre docencia (37 \%), justo antes del ODS 13: Acción por el clima.

$2 \quad$ La Meta 4.7 del ODS 4 establece: Para 2030, garantizar que todos los alumnos adquieran los conocimientos teóricos y prácticos necesarios para promover el desarrollo sostenible, entre otras cosas mediante la educación para el desarrollo sostenible y la adopción de estilos de vida sostenibles, los derechos humanos, la igualdad entre los géneros, la promoción de una cultura de paz y no violencia, la ciudadanía mundial y la valoración de la diversidad cultural y de la contribución de la cultura al desarrollo sostenible, entre otros medios. 
Tabla 3 .

Distribución de los ODS por categorías del trabajo realizado

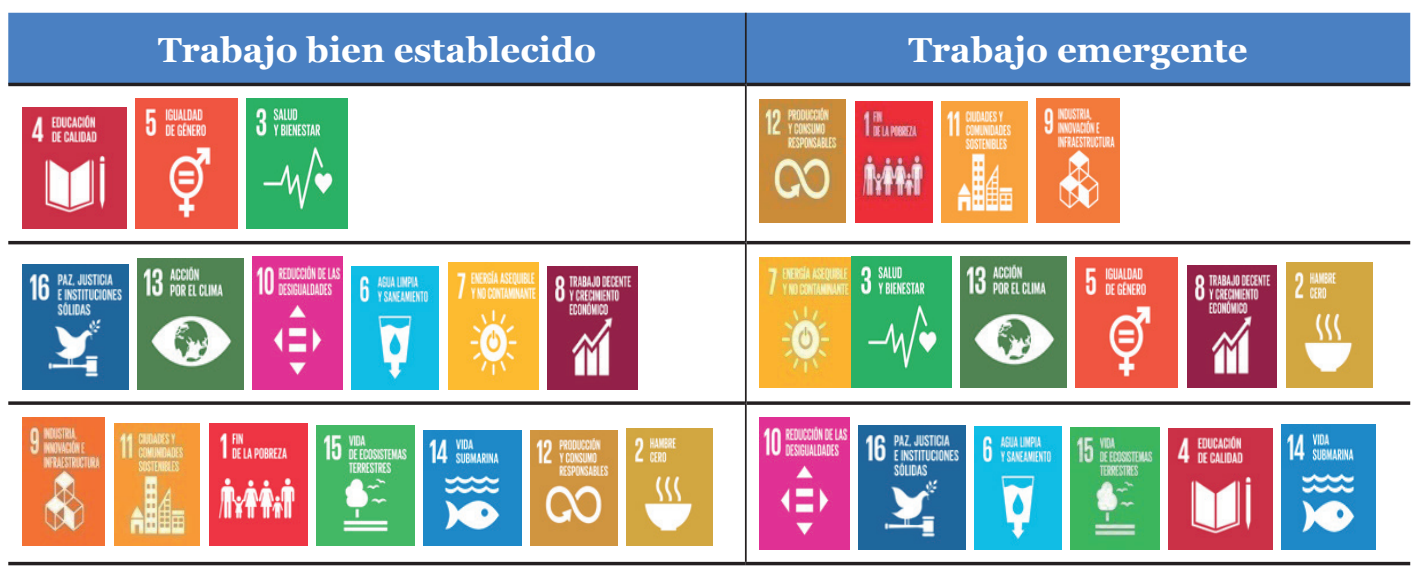

Fuente: Elaboración propia a partir de Encuesta IAU (2020).

Para comprender cómo se realiza la integración de los temas sobre el desarrollo sostenible en la docencia se analizan las respuestas a dos preguntas de la encuesta que se centraron en indagar acerca de los cursos ofrecidos en las IES. Una de las preguntas indagó sobre cursos específicos (por ejemplo, títulos de maestría en desarrollo sostenible) y la otra, se centró en cursos que integran el desarrollo sostenible (por ejemplo, un curso de métodos de ciencias sociales que utiliza el desarrollo sostenible).

Los resultados arrojaron que el $65 \%$ de las instituciones encuestadas, proveen cursos nuevos sobre desarrollo sostenible o integran el tema en los ya existentes. Un $14 \%$ expresó que no existían cursos de este tipo y un 21 \% que no conocían de la existencia de curso centrados en sostenibilidad. Especialmente, personal administrativo no estaban al tanto de dichos cursos y contenidos, de lo que se infiere que las IES no integran el desarrollo sostenible en sus planes y programas institucionales, como parte del enfoque institucional integrado (IAU, 2020).

También se reporta que aun cuando más del $80 \%$ de las respuestas indicaron haber incorporado el desarrollo sostenible en su enseñanza, solo el $65 \%$ dijo que era parte de un curso o programa específico. Este es un resultado inquietante, señalado en el informe que podría significar que el desarrollo sostenible es parte formal del proceso de enseñanza y aprendizaje, pero que no necesariamente forma parte del plan de estudios oficial. Otra razón para la diferencia en las respuestas podría ser también, que las personas entendieron las preguntas de manera diferente y respondieron más de forma subjetiva, lo cual pudiese, entonces significar que mientras el $80 \%$ quiere que el desarrollo sostenible sea parte del proceso formativo formal, solo el $65 \%$ realmente tiene cursos específicos para ello.

\subsection{Reporte 2019 del Acuerdo SDG}

El Acuerdo SDG es una iniciativa internacional desarrollada por la Alianza Global de un grupo de redes de sostenibilidad de universidades e instituciones de educación superior de distintas partes del mundo y la EAUC (por sus siglas en inglés Asociación Ambiental para Universidades y Colegios) con el fin de promover el papel fundamental que tiene la educación superior en la entrega de los ODS y divulgar las mejores prácticas, del compromiso del sector de educación terciaria en el cumplimiento de estos objetivos globales. 
Esta iniciativa es respaldada por Higher Education Sustainability Initiative (HESI) de la ONU y muchos otros socios globales. Fue lanzada en 2017 y actualmente está integrada por 110 instituciones, 103 organizaciones de apoyo y 817 individuos, todos distribuidos en 85 países. Los firmantes del Acuerdo SDG se han comprometido con la incorporación de los SDG en sus actividades de educación, investigación, liderazgo, operaciones, administración y participación y, para ello, deben informar sobre su progreso hacia los ODS. (SDG Accord, 2019).

El reporte fue elaborado con base en 51 instituciones, de las 110 IES participantes signatarios del Acuerdo SDG, que respondieron efectivamente a la encuesta, informando sobre sus respectivos trabajos con los ODS. Las informaciones y datos que se observan en el reporte corresponden a 19 países, y tres cuartas partes son universidades británicas, lo cual sugiere que su alcance no es totalmente global (SDG Accord, 2019).

Los principales hallazgos de este reporte muestran que el $70 \%$ de las instituciones encuestadas asocian sus actividades a los ODS parcial o totalmente y la mayoría de las instituciones ( $71 \%$ ) tienen a los ODS como una prioridad estratégica.

De acuerdo con este reporte los ODS en los cuales las instituciones han tenido mayor impacto son: el ODS 4: Educación de calidad, ODS 3: Buena salud y bienestar, y sobre el ODS 5: Igualdad de género. Y las prioridades de trabajo las han focalizado así: el $72 \%$ indicó direccionar hacia el ODS 13: Acción por el clima, el 70 \% hacia el ODS 4: Educación de calidad y el $58 \%$ hacia el ODS 3: Buena salud y bienestar. En cuanto al otro extremo del espectro, es decir, los ODS en los que las instituciones consideraron que tuvieron un menor impacto indican que fueron: los ODS 1 Cero pobreza, ODS 2 Hambre cero y el ODS 6 Agua limpia y saneamiento.

Tabla 4.

Distribución de los ODS según impacto del trabajo realizado

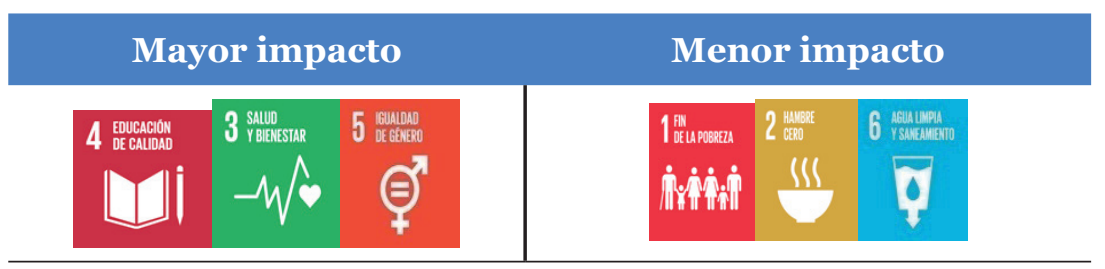

Fuente: Elaboración propia a partir de SDG Accord, 2019.

En relación al trabajo que las IES encuestadas reportan de forma específica por cada ODS, se indica que el ODS 4: Educación de calidad, continúa siendo de gran importancia, como se puede esperar de las instituciones educativas, pero la Acción por el clima (ODS 13) lo ha superado en términos de priorización.

Sobre la integración de los ODS en la docencia y la investigación se reportan avances. El 69 \% de las instituciones comunican que han encontrado formas innovadoras de aumentar la capacidad del personal y los estudiantes para abordar los ODS, estos van desde aplicaciones de gamificación, hasta incentivos al voluntariado estudiantil, creando campeones de sostenibilidad en cada departamento del personal.

De igual manera se reporta que un 49 \% de las IES respondieron que han realizado en forma parcial actualizaciones e incorporación de los ODS en los procesos institucionales, tales como estrategias, políticas, actualizaciones del currículo o procesos de revisión de cursos. 


\subsection{Referencias a experiencias como casos de estudio.}

Otro de los avances significativos del proceso de incorporación, integración o también llamado alineamiento institucional de los ODS a la docencia son las acciones y actividades de integración de estos Objetivos Globales a los planes de estudio de las titulaciones oficiales que, como acción formativa, se realiza en las IES. Aún queda pendiente constatar si han emergido o no nuevas titulaciones o programas de estudios en materia de sostenibilidad, así como la revisión de las áreas, contenidos y formas de vinculación con los 17 ODS y la exploración de su evolución y avances hacia el futuro.

Pero, este examen amerita de un mapeo profundo y exhaustivo de esas acciones y actividades y, por consiguiente, un proceso de intervención de IES comprometidas con los ODS que documenten sus experiencias o iniciativas en ese sentido. Desafortunadamente, aún no ha sido posible alcanzar ese desafío, aun cuando ya hay universidades e IES que han iniciado a mapear sus acciones y actividades relacionadas con los ODS, tal como antes se mencionó que lo ha referenciado la IAU (2020).

No obstante, el impacto de los ODS sobre la emergencia y la transformación de las titulaciones se empieza a notar en algunos casos que pueden ser considerados paradigmáticos, aunque, todavía muy reducidos en número (Nhamo y Mjimba, 2020; Shava et al, 2020; Kanapathy et al, 2019), pueden ser referenciados como casos de estudio de experiencias o iniciativas de educación para los ODS (Miñano y García, 2020) considerados, algunos como ejemplos icónicos. (Tabla 5).

En la Matriz de iniciativas de integración de los ODS en las acciones docentes (Tabla 5) se observan avances con respecto a la integración de estos Objetivos a la docencia formal o acciones de integración global de los ODS en el curriculum. Se observan distintos ámbitos de actuación que van desde la incorporación de estos objetivos en las asignaturas existentes, así como acciones de actualización de contenidos contextualizándolos de acuerdo con los perfiles académicos y disciplinarios que en las distintas titulaciones de grado o postgrado se ofrecen en las universidades del mundo.

La mayoría de estas experiencias o iniciativas realizan integración de los ODS en asignaturas obligatorias transversales abordando temas de desarrollo sostenible y en específico acerca de la Agenda 2030 y los ODS; es decir, realizan acciones de transversalización de los ODS en los planes de estudio.

También es importante mencionar que muchas de estas experiencias, no solamente abarcan la inclusión de contenidos de sostenibilidad en el temario de las distintas asignaturas, sino que promueven además, cambios más globales en la concepción del proceso educativo y de los procesos de enseñanza y aprendizaje (Aznar et.al, 2016; BautistaCerro y González, 2017; Leal Filho, 2018; Evans, 2019), los cuales están siendo mapeados como casos de aprendizaje por servicio, así como iniciativas formativas y de gestión que implican a toda la comunidad universitaria e iniciativas abiertas y en colaboración con otros sectores sociales recopiladas por la REDS en su dosier de casos inspiradores de educación para los ODS en las universidades españolas (Miñano y García, 2020).

\section{A modo de conclusión}

En términos generales encontramos que tanto en el informe de la IAU como en el reporte del Acuerdo SDG se aprecia un aumento del compromiso de la educación superior con los ODS y cómo las IES están trabajando cada vez más en y para el desarrollo 
sostenible. Con ambos informes se constata que el área de la enseñanza y el aprendizaje se constituyen en la dimensión de la educación superior donde se vislumbra el mayor potencial para incorporar el desarrollo sostenible, aun cuando esto no necesariamente se traduce en el cambio necesario para alcanzar los Objetivos Globales.

Aun cuando las encuestas sobre las que se basaron ambos informes fueron realizadas con participantes distintos, ambos grupos de participantes coinciden en identificar que los tres ODS más trabajados o clasificados en «trabajo bien establecido» o «trabajo con mayor impacto» son el ODS4: Educación de Calidad, ODS5: Igualdad de Género y el ODS3: Salud y Bienestar, difiriendo en la proporción de respuestas que dichos ODS obtuvieron en las respectivas encuestas.

Como se puede apreciar los resultados de las encuestas analizadas aun cuando, la de la AIU, más que la del Acuerdo Global por los ODS, arrojan informaciones valiosas a cerca de lo que está sucediendo en la educación superior y la investigación para el desarrollo sostenible a nivel mundial y mostrar que las universidades de todo el mundo están trabajando en alguna medida en todos los ODS, así como evidenciar dimensiones en cuanto a cómo unos objetivos son abordados con mayor preferencia que otros e identificar en forma global, áreas de trabajo prioritarias en cada IES, aun así no permite apreciar acciones particulares y especificas respecto avances en cuanto a las titulaciones y planes de estudio relacionados tangencial o específicamente con los ODS.

La matriz de iniciativas de integración de los ODS en las acciones docentes facilitó mapear la actuación de algunas IES que mayoritariamente realizan estas acciones a través de procesos de rediseños específicos de asignaturas o cursos mediante los cuales abordan los ODS en forma global. Muy pocas iniciativas envuelven procesos formales de integración curricular o también llamado transversalización o sostenibilización curricular. Lo que indica que estas experiencias continúan siendo aún, muchos más esfuerzos individuales de cada docente, que resultados de políticas institucionales. No obstante, son iniciativas que bien pueden considerarse inspiradoras o una buena práctica de referencia válida para otros actores y otros contextos.

Para profundizar y conocer tanto factores del proceso de integración, los ámbitos y modalidades del accionar de estas y otras iniciativas existente, y no reportadas en este artículo; así como detalles del proceso de integración explícita de los ODS en todo los plan de estudios o en los cursos/asignaturas, así como la aplicabilidad a los escenarios cotidianos como tributo de graduación para estudiantes, se hace imprescindible contar con información más explícita acerca de esto, por ello, replicar experiencias como la desarrollada por REDS, así como hacer disponible plataformas en línea en las cuales los docentes expongan sus experiencias de rediseños curriculares e incorporación a los planes de estudios de los ODS y se mapeen asimismo las nuevas titulaciones que vienen emergiendo. 


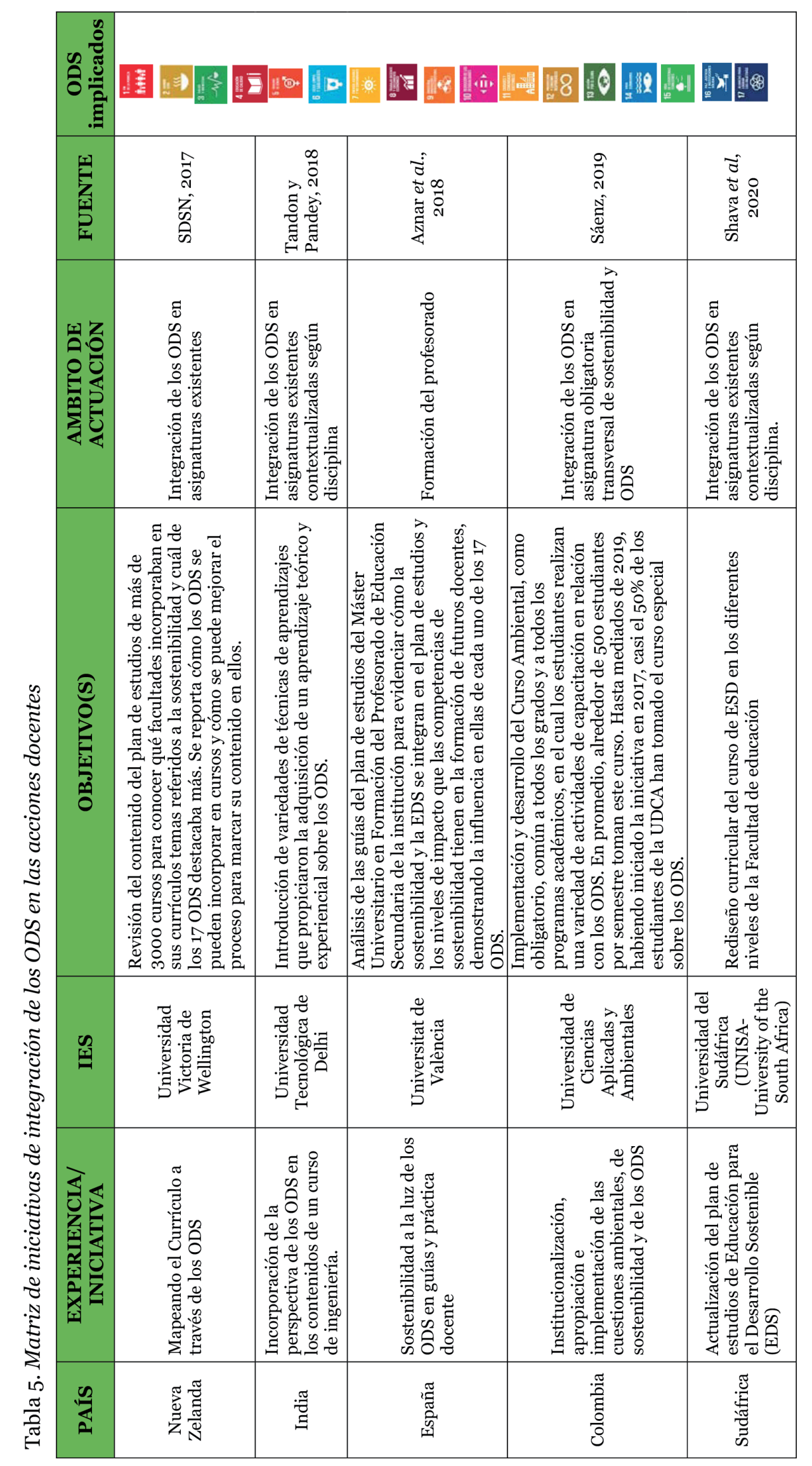




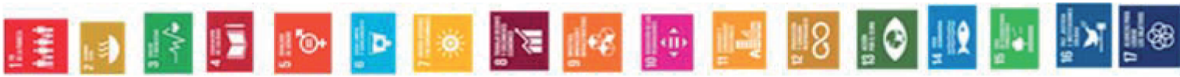

\begin{tabular}{|c|c|c|c|c|}
\hline 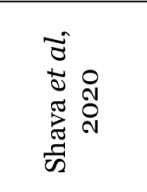 & 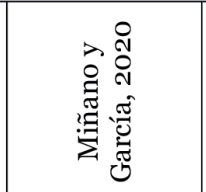 & 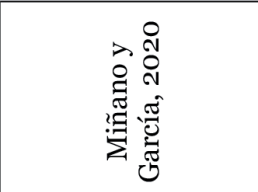 & 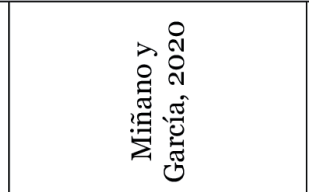 & 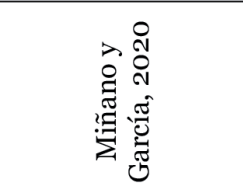 \\
\hline 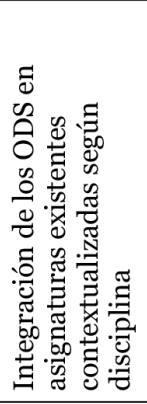 & 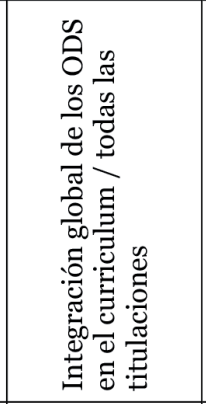 & 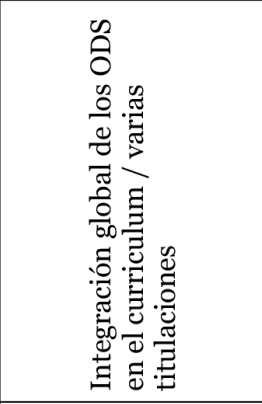 & 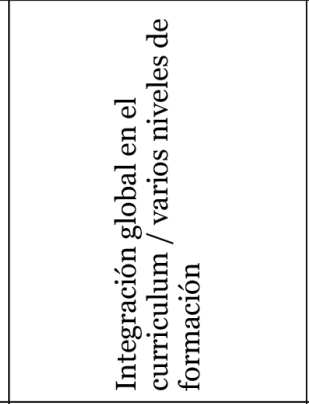 & 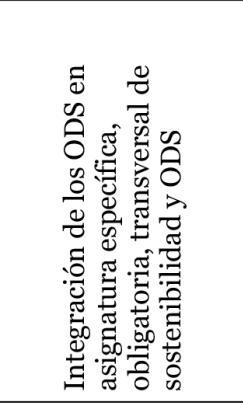 \\
\hline 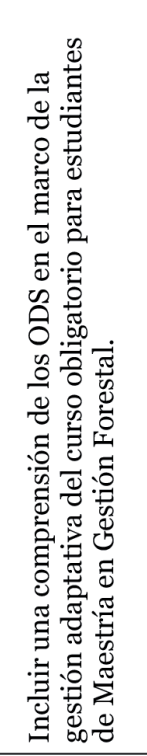 & 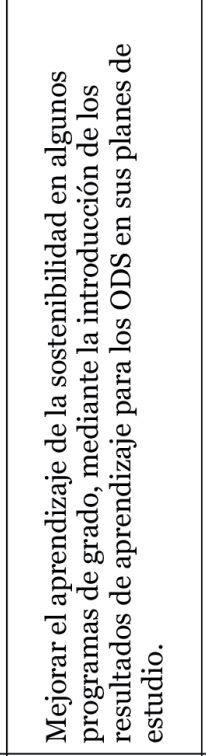 & 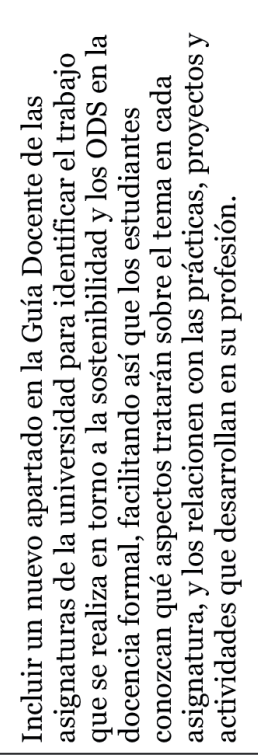 & 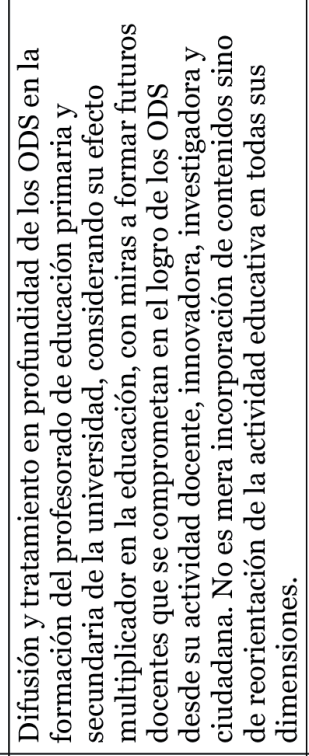 & 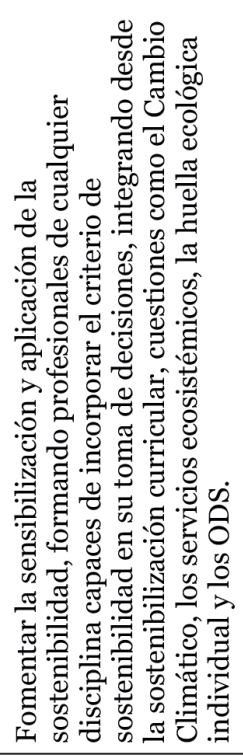 \\
\hline 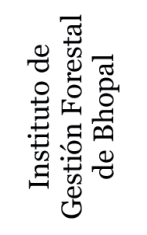 & 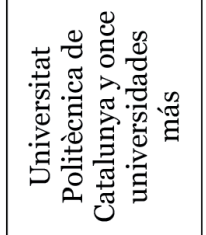 & 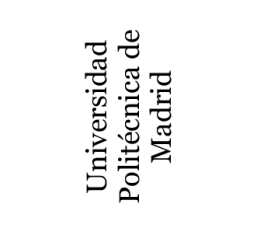 & 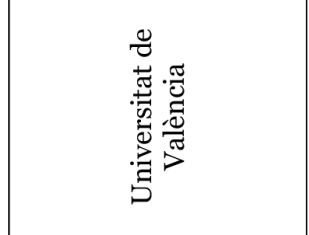 & 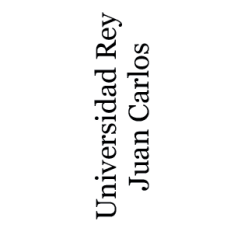 \\
\hline 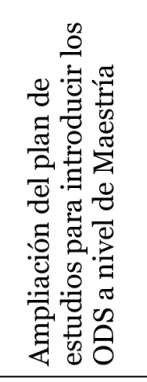 & 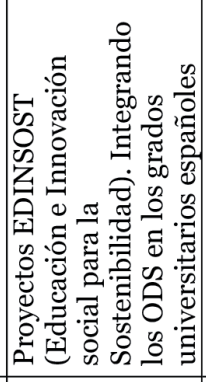 & 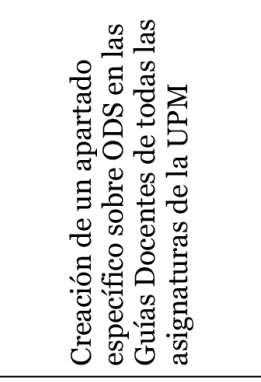 & 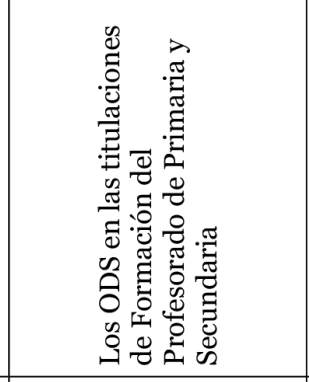 & 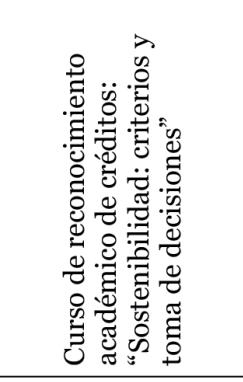 \\
\hline : & 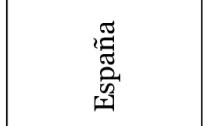 & 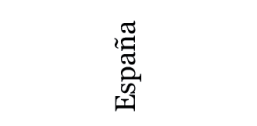 & $\begin{array}{l}\text { प्च } \\
\text { 空 }\end{array}$ & 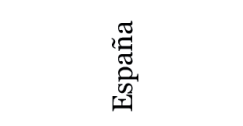 \\
\hline
\end{tabular}




\section{Referencias}

Albareda, S., García-González, E., Jiménez-Fontana, R., Solís-Espallargas, C. (2019). Implementing Pedagogical Approaches for ESD in Initial Teacher Training at Spanish Universities. Sustainability Journal. 11(18).

Antúnez, M. (2017). La problemática del proceso de sostenibilización curricular en el contexto universitario español: La formación del profesorado como catalizador. Tesis doctoral por la Universidad de Córdoba.

AntúnezM., Gomera A.yVillamandos, F.(2017). SostenibilidadyCurrículum: Problemática y posibles soluciones en el contexto universitario español. Profesorado. Revista de Currículum y Formación de Profesorado, 21(4), 197-214.

Antúnez, M., Gomera, A., Vaquero, M.y Villamandos, F. (2018). Percepción del profesorado universitario sobre la Agenda 2030: el caso de la Universidad de Córdoba. Libro de resúmenes del IV Congreso Internacional de Estudios del Desarrollo (p. 108). Córdoba, España: Universidad Loyola.

Aznar Mínguet, P.; Ull, M. A.; Piñero, A. y Martínez-Agut, M. P. (2016). Competencies for Sustainability in the Curricula of all new Degrees from the University of Valencia (Spain). En: Barth, M.; Michelsen, G.; Rieckmann, M. y Thomas, I. (eds.) Handbook of Higher Education for Sustainable Development. New York, Routledge.

Bautista-Cerro R., M., y Díaz G., M. (2017). La sostenibilidad en los grados universitarios: presencia y coherencia. Teoría de la Educación. Revista Interuniversitaria, 29 [1 (en-jun)], pp. 161-187.

Cubero, J. (2019). Integración de los ODS y las competencias transversales en el ámbito universitario. Forum calidad, Año 29, No 298, 2019, pp. 44-53.

El-Jardali, F., Ataya, N., y Fadlallah, R. (2018). Changing roles of universities in the era of SDGs: rising up to the global challenge through institutionalising partnerships with governments and communities. Health Research Policy Systems, 16(38).

Evans, T.L. (2019). Competencies and Pedagogies for Sustainability Education: A Roadmap for Sustainability Studies Program Development in Colleges and Universities. Sustainability, 11(19), 5526.

García-González, E. et al. (2019). Inclusion of Sustainability in University Classrooms Through Methodology. En. W. Leal Filho, et al. (eds.), Handbook of Theory and Practice of Sustainable Development in Higher Education, World Sustainability Series.

Gough G. y Longhurst J. (2018). Monitoring Progress Towards Implementing Sustainability and Representing the UN Sustainable Development Goals (SDGs) in the Curriculum at UWE Bristol. En: Leal Filho, W. (eds) Implementing Sustainability in the Curriculum of Universities. World Sustainability Series. Springer, Cham. 
GUNi. (2019). Implementing the 2030 Agenda at Higher Education Institutions: Challenges and Responses. Global University Network for Innovation.

HESI. (2019). Progress towards the Global Goals in the University and College sector. Annual SDG Accord Report 2019. Higher Education Sustainability Initiative. New York.

IAU. (2020). Higher Education and the 2030 Agenda: Moving into the 'Decade of Action and Delivery for the SDGs'. Paris: International Association of Universities (IAU) / International Universities Bureau.

Instituto Internacional de la UNESCO para la Educación Superior en América Latina y el Caribe (2020). Contribución de la educación superior a los Objetivos de Desarrollo Sostenible: marco analítico. Caracas, UNESCO-IESALC. Disponible en: https://www.iesalc.unesco.org/2020/02/17/2595/

Kanapathy, S., Lee, K., Sivapalan, S., Mokhtar, M., Syed Zakaria, S. y Mohd Zahidi, A. (2019). "Sustainable development concept in the chemistry curriculum: An exploration of foundation students perspective", International Journal of Sustainability in Higher Education Vol. 20 No. 1, pp. 2-22.

Kosta K. (2018). Sustainability Curriculum in UK University Sustainability Reports. En: Leal Filho, W. (eds). Implementing Sustainability in the Curriculum of Universities. World Sustainability Series. Springer, Cham.

Leal Filho, W. , Shiel, Ch., Paço, A., Mifsud, M., Ávila, L., Brandli, L., MolthanHill, P., Pace, P., Azeiteiro, U., Vargas, V. y Caeiro, S. (2019). Sustainable Development Goals and sustainability teaching at universities: Falling behind or getting ahead of the pack? Journal of Cleaner Production. 232. 10.1016/j. jclepro.2019.05.309.

Lozano, R., Merrill, M., Sammalisto, K., Ceulemans, K., y Lozano, F. (2017). Connecting Competences and Pedagogical Approaches for Sustainable Development in Higher Education: A Literature Review and Framework Proposal. Sustainability, 9(10), 1889. doi:10.3390/su9101889

McCowan, T. (2019). Higher Education For and Beyond the Sustainable Development Goals. London: Palgrave Macmillan.

Mindt, L. y Rieckmann, M. (2017). Developing competencies for sustainability-driven entrepreneurship in higher education: A literature review on teaching and learning methods. Revista interuniversitaria. 29. 129-159.

Miñano, R. y García H., M. (Editores) (2020). Implementando la Agenda 2030 en la universidad. Casos inspiradores, Madrid: Red Española para el Desarrollo Sostenible (REDS).

Murga-Menoyo, M. A., y Novo, M. (2015). The Processes of Integrating Sustainability in Higher Education Curricula: A Theoretical-Practical Experience Regarding Key Competences and Their Cross-Curricular Incorporation into Degree Courses. En Leal Filho, W. (Ed.), Transformative Approaches to Sustainable Development at Universities (pp. 119-135). Cham: Springer International Publishing. 
Ng, A.W., Leung, Tiffany C.H y Lo, J. M.K. (2019). Developing Sustainability Competence for Future Professional Accountants: The Integrative Role of an Undergraduate Program. En. W. Leal Filho et al. (eds.), Handbook of Theory and Practice of Sustainable Development in Higher Education, World Sustainability Series, doi 10.1007/978-3-319-47868-5_1

Nhamo, G. y Mjimba, V. (Eds). (2020). Sustainable Development Goals and Institutions of Higher Education. Sustainable Development Goals Series. Springer International Nature Switzerland.

Paletta, A., Bonoli, A. (2019). "Governing the university in the perspective of the Univet Nations 2030 Agenda. The case of the University of Bologna”, International Journal of Sustainability in Higher Education, 2O(3), pp. 500-514.

Reickman, M. (2018). Learning to transform the world: Key competencies in ESD. In Issues and Trends in Education for Sustainable Development; Leicht, J., Byun, W.J., Eds.; United Nations Educational, Scientific and Cultural Organization (UNESCO): Paris, France, 2018; pp. 39-59.

Sánchez C., M. F. y Murga-Menoyo, M. A. (2019). Place-Based Education: una estrategia para la sostenibilización curricular de la educación superior, Bordón, Revista de Pedagogía, 71(2), 2019, 155-174.

Scarff S., C. y Ceulemans, K. (2017). Teaching Sustainability in Higher Education: Pedagogical Styles that Make a Difference, Canadian Journal of Higher Education Revue/ canadienne d'enseignement supérieur, CJHE / RCES, Volume 47, No. 2, 2017, pp. 47 - 70.

SDG Accord. (2019). Annual SDG Accord Report 2019 Progress towards the Global Goals in the University and College sector. EAUC.

SDSN Australia/Pacific. (2017). Getting started with the SDGs in universities: A guide for universities, higher education institutions, and the academic sector. Australia, New Zealand and Pacific Edition. Melbourne: Sustainable Development Solutions Network - Australia/Pacific.

Sáenz, O. (2019). Implementation of the Sustainable Development Goals (SDGs) in Higher Education Institutions: Recommendations Based on the Experience of a Latin American University. En: Implementing the 2030 Agenda at Higher Education Institutions: Challenges and Responses. Global University Network for Innovation.

Segalàs, J., y Sánchez, F. (2019). El proyecto EDINSOST. Formación en las Universidades españolas de profesionales como agentes de cambio para afrontar los retos de la sociedad. Revista de Educación Ambiental y Sostenibilidad, 1(1), 1204.

Serrate González, S., Lucas, J., Caballero, D. y Muñoz-Rodríguez, J. (2019). Responsabilidad universitaria en la implementación de los objetivos de desarrollo sostenible. European Journal of Child Development, Education and Psychopathology. [S.1.], v. 7, n. 2, p. 183-196, nov. 2019. 
Shava S., Makokotlela M.V., Hebe H. (2020). Role of SDGs in Reconceptualising the Education for Sustainable Development Curriculum in Higher Education in South Africa. En: Nhamo G., Odularu G., Mjimba V. (eds) Scaling up SDGs Implementation. Sustainable Development Goals Series. Springer, Cham.

Sulitest. (2019). Raising and Mapping Awareness of the Global Goals.

Tandon R. y Pandey, P. (2019). Disciplines, Professions and Sustainable Development Goals (SDGs): Challenges in Higher Education in India. En: Implementing the 2030 Agenda at Higher Education Institutions: Challenges and Responses. Global University Network for Innovation.

UNESCO. (2014). Unesco roadmap for implementing the Global Action Programme on Education for Sustainable Development. Disponible en: https://unesdoc. unesco.org/ark:/48223/pfoooo230514

UNESCO. (2015). Education 2030: Incheon Declaration and Framework for Action for the implementation of Sustainable Development Goal 4: Ensure inclusive and equitable quality education and promote lifelong learning opportunities for all.

UNESCO. (2017). Educación para los Objetivos de Desarrollo Sostenible. Objetivos de aprendizaje. [Tabla]. Recuperado de: https://unesdoc.unesco.org/ark:/48223/ pfoooo247444

UNESCO. (2018). Global Education Monitoring Report 2017/18. Paris: UNESCO.

Valderrama-Hernández, R., Alcántara, L., Sánchez-Carracedo, F., Caballero, D., GilDoménech, D., Serrate, S., Vidal-Raméntol, S., y Miñano, R. (2019). ¿Forma en sostenibilidad el sistema universitario español? Visión del alumnado de cuatro universidades. Educación XX1, 22(1), 1-26. 